\title{
ACCURATE GRADIENT COMPUTATIONS AT INTERFACES USING FINITE ELEMENT METHODS
}

\author{
FANGFANG QIN $^{a}, Z_{\text {HAOHUI WANG }}{ }^{b}, \mathrm{ZHIJIE} \mathrm{MA}^{c}, d, \mathrm{ZHILIN} \mathrm{LI}^{b, *}$ \\ ${ }^{a}$ School of Science \\ Nanjing University of Posts and Telecommunications, Nanjing, Jiangsu, 210023 China \\ e-mail: qinfangfangmatha163.com \\ ${ }^{b}$ Department of Mathematics \\ North Carolina State University, Raleigh, NC 27695, USA \\ e-mail: zwang24@ncsu.edu, zhilin@math.ncsu.edu \\ ${ }^{c}$ College of Resource and Environment \\ Wuhan University of Technology, Wuhan, 430070 China \\ ${ }^{d}$ China Institute of Water Resource and Hydropower Research (IWHR), Beijing, 100038 China \\ e-mail: mazj88@126.com
}

\begin{abstract}
New finite element methods are proposed for elliptic interface problems in one and two dimensions. The main motivation is to get not only an accurate solution, but also an accurate first order derivative at the interface (from each side). The key in 1D is to use the idea of Wheeler (1974). For 2D interface problems, the point is to introduce a small tube near the interface and propose the gradient as part of unknowns, which is similar to a mixed finite element method, but only at the interface. Thus the computational cost is just slightly higher than in the standard finite element method. We present a rigorous one dimensional analysis, which shows a second order convergence order for both the solution and the gradient in 1D. For two dimensional problems, we present numerical results and observe second order convergence for the solution, and super-convergence for the gradient at the interface.
\end{abstract}

Keywords: elliptic interface problems, gradient/flux computation, IFEM, mixed FE formulation, computational tube.

\section{Introduction}

In this paper, we consider the following interface problems:

$-\nabla \cdot(\beta(\mathbf{x}) \nabla u(\mathbf{x}))+q(\mathbf{x}) u(\mathbf{x})=f(\mathbf{x}), \quad \mathbf{x} \in \Omega \backslash \Gamma$,

in one and two dimensions. We assume that there is a closed interface $\Gamma$ in the solution domain across which the coefficient $\beta$ has a finite jump (discontinuity),

$$
\beta(\mathbf{x})= \begin{cases}\beta_{1} & \text { if } \mathbf{x} \in \Omega_{1}, \\ \beta_{2} & \text { if } \mathbf{x} \in \Omega_{2} .\end{cases}
$$

Because of the discontinuity, the natural jump condition should be satisfied, that is, both the solution and the flux

\footnotetext{
${ }^{*}$ Corresponding author
}

should be continuous across the interface $\Gamma$,

$$
[u]_{\Gamma}=0, \quad\left[\beta \frac{\partial u}{\partial n}\right]_{\Gamma}=0,
$$

where the jump at a point $\mathbf{X}=(X, Y)$ on the interface $\Gamma$ is defined as

$$
\begin{aligned}
{\left[\beta \frac{\partial u}{\partial n}\right]_{\mathbf{X}}=} & \lim _{\mathbf{x} \rightarrow \mathbf{X}, \mathbf{x} \in \Omega_{2}} \beta(\mathbf{x}) \frac{\partial u(\mathbf{x})}{\partial n} \\
& -\lim _{\mathbf{x} \rightarrow \mathbf{X}, \mathbf{x} \in \Omega_{1}} \beta(\mathbf{x}) \frac{\partial u(\mathbf{x})}{\partial n}
\end{aligned}
$$

and

$$
u_{n}=\mathbf{n} \cdot \nabla u=\frac{\partial u}{\partial n}
$$

is the normal derivative of solution $u(\mathbf{X})$, and $\mathbf{n}$ is the unit normal direction pointing to $\Omega_{2}$ side; see Fig. 1 for 
an illustration. Since a finite element discretization is used, we assume that $f(\mathbf{x}) \in L^{2}\left(\Omega_{i}\right), q(\mathbf{x}) \in L^{\infty}\left(\Omega_{i}\right)$ excluding $\Gamma$. For regularity we also assume that $\beta(\mathbf{x}) \geq$ $\beta_{0}>0$ and $q(\mathbf{x}) \geq 0 ; \Gamma \in C^{1}$. From these assumptions, we know that the solution $u(\mathbf{x}) \in H^{2}\left(\Omega_{i}\right)$ for $i=1,2$.

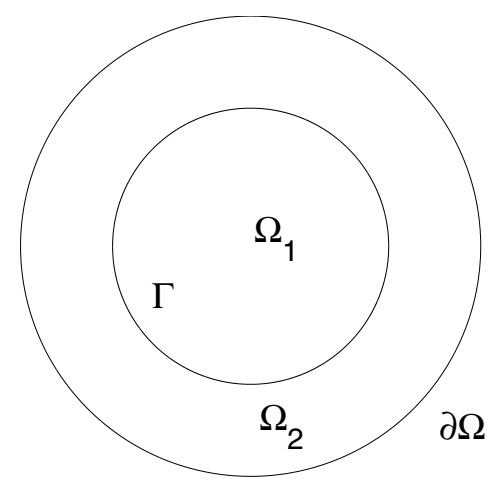

Fig. 1. Diagram of domains $\Omega_{1}$ and $\Omega_{2}$, an interface $\Gamma$, and the boundary $\partial \Omega$.

There are many applications of such an interface problem; see, e.g., the works of Sutton and Balluffi (1995), Zienkiewicz and Taylor (2000), Li and Ito (2006) or Kwak and Chang (2010) and the references therein. Many numerical methods have been developed for solving such an important problem. For the elliptic interface problem (1)-(3), the solution has a low global regularity. Thus, a direct conforming finite element method based on polynomial basis functions over a mesh likely works poorly if the mesh is not aligned along the interface, since the FE solution is a smooth piece in an element and cannot capture the discontinuity in the direction at the interface. Nevertheless, it is reasonable to assume that the solution is piecewise smooth excluding the interface. For example, if the coefficient is a piecewise constant in each sub-domain, then the solution in each sub-domain is an analytic function in the interior, but has a jump in the normal derivative of the solution across the interface from PDE limiting theory (Kevorkian, 1990). The gradient used in this paper is defined as the limiting gradient from each side of the interface.

Naturally, finite element methods can be and have been applied to solve interface problems. It is well known that a second order accurate approximation to the solution of an interface problem can be generated by the Galerkin finite element method with the standard linear basis functions if the triangulation is aligned with the interface, that is, a body fitted mesh is used (see, e.g., Babuška, 1970; Bramble and King, 1996; Chen and Zou, 1998; Xu, 1982; Karczewska and Boguniewicz, 2016). Other state of art methods include the IGA-FEM, an open source 3D
Matlab Isogeometric Analysis package (Anitescu, 2017), or DPG, a discontinuous Petrov-Galerkin finite element method (Carstensen and Weggler, 2014).

Some kind of posterior techniques or at least quadratic elements is needed in order to get a second order accurate gradient from each side of the interface. The cost in mesh generation coupled with an unstructured linear solver makes the body-fitted mesh approach less competitive.

Alternatively, we can use a structured mesh (non-body fitted) to solve such an interface problem. There are also quite a few finite element methods using Cartesian meshes. The immersed finite element method (IFEM) was developed for 1D and 2D interface problems by $\mathrm{Li}$ (1998) as well as $\mathrm{Li}$ and Wu (2003), respectively. Since then, many IFEMs and related analysis have appeared in the literature (see, e.g., Chou and Wee, 2010; He and Lin, 2011; Ji and Li, 2016), along with applications (An and Chen, 2014; Lin and Zhang, 2012; 2015, Yang, 2002). Often they provide a second order accurate solution in the $L^{2}$ norm but only a first order accurate flux.

Nevertheless, in many applications the primary interest is focused on flux values at interfaces in addition to solutions of the governing differential equations (see, e.g., Chou, 2012; Douglas Jr and Wheeler, 1974; Wheeler, 1974). Most numerical methods for interface problems based on structure meshes are between first and second order accurate for the solution, but the accuracy for the gradient is usually one order lower. Note that the gradient recovering techniques (e.g., Wahlbin, 1995; Zhang and Naga, 2005) hardly work for structured meshes because of the arbitrariness of the interface and the underlying mesh. The mixed finite element approach and a few other methods that can find accurate solutions and gradients simultaneously in the entire domain often lead to saddle problems and are computationally expensive, and so are not ideal choices if we are only interested in an accurate gradient near an interface or a boundary. We note that, very recently, superconvergence of the IFEM has been studied for 1D interface problems (Cao and Zhang, 2017). In this paper, we develop two new finite element methods; one is in $1 \mathrm{D}$, the other one is $2 \mathrm{D}$, for obtaining accurate approximations of the flux values at interfaces.

The rest of the paper is organized as follows. In Section 2, we explain the one dimensional algorithm and provide its theoretical analysis. We explain how to construct approximations to the flux values at the left and right of the interface, and approximations to the flux values at the boundary of the domain. We also provide a numerical example. The numerical algorithm for two dimensional problems is explained in Section 3 along with some numerical experiments. We conclude the paper in Section 4. 


\section{One-dimensional algorithm and analysis}

The one dimensional model problem has the following form:

$$
\begin{aligned}
-\left(\beta(x) u^{\prime}(x)\right)^{\prime}+q(x) u(x) & =f(x), \\
u(0)=0, \quad u(1) & =0,
\end{aligned}
$$

where $0<x<1, \beta$ is a piecewise constant and has a finite jump at an interface $0<\alpha<1$, and a homogenous boundary condition for simplicity of the discussion. Across the interface, the natural jump conditions are

$$
[u]_{\alpha}=0, \quad\left[\beta u^{\prime}(x)\right]_{\alpha}=0 .
$$

We define the standard bilinear form,

$$
\begin{array}{r}
a(u, v) \\
=\int_{0}^{1}\left(\beta(x) u^{\prime}(x) v^{\prime}(x)+q(x) u(x) v(x)\right) \mathrm{d} x, \\
\forall u(x), v(x) \in H_{0}^{1}(0,1),
\end{array}
$$

where $H_{0}^{1}(0,1)$ is the Sobolev space (Brenner and Scott, 2007; Adams and Fournier, 2003; Tartar, 2007):

$$
\begin{aligned}
& H_{0}^{1}(0,1) \\
& \quad=\left\{v(x) \in H^{1}(0,1) \text { and } v(0)=v(1)=0\right\} .
\end{aligned}
$$

The solution of the differential equation $u(x) \in H_{0}^{1}(0,1)$ is also the solution of the following variational problem:

$$
\begin{aligned}
a(u, v)=(f, v) & =\int_{0}^{1} f(x) v(x) \mathrm{d} x, \\
& \forall v(x) \in H_{0}^{1}(0,1) .
\end{aligned}
$$

Integration by parts over the disjoint intervals $(0, \alpha)$ and $(\alpha, 1)$ yields

$$
\begin{aligned}
0= & \int_{0}^{\alpha}\left\{-\left(\beta u^{\prime}\right)^{\prime}+q u-f\right\} v \mathrm{~d} x+\beta_{1} u_{x}^{-} v^{-} \\
& +\int_{\alpha}^{1}\left\{-\left(\beta u^{\prime}\right)^{\prime}+q u-f\right\} v \mathrm{~d} x-\beta_{2} u_{x}^{+} v^{+} .
\end{aligned}
$$

The superscripts - and + indicate the limiting value as $x$ approaches $\alpha$ from the left and right, respectively, and $u_{x}=u^{\prime}$. Recall that $v^{-}=v^{+}$for any $v$ in $H_{0}^{1}$. It follows that the differential equation holds in each interval and that

$$
[u]=u^{+}-u^{-}=0, \quad\left[\beta u_{x}\right]=\beta^{+} u_{x}^{+}-\beta^{-} u_{x}^{-}=0,
$$

where we have drop the subscript $\alpha$ in the jumps for simplicity. These relations are the same as in (4), which indicates that the discontinuity in the coefficient $\beta(x)$ does not cause any additional difficulty for the theoretical analysis of the finite element method. The weak solution will satisfy the jump conditions (5).
2.1. Immersed finite element method in 1D. Now we briefly explain the immersed finite element method (IFEM) in 1D introduced by Li (1998). As in the IFEM, we use a uniform grid, i.e., $x_{i}=i h, i=0,1, \ldots, n$, and assume that $\alpha \in\left(x_{j}, x_{j+1}\right)$. Since it is a one dimensional problem, we use $\beta^{+}$for $\beta_{2}, \beta^{-}$for $\beta_{1}$, and so on.

If the interface does not cut an interval $\left(x_{i}, x_{i+1}\right)$, then we use the standard piecewise linear basis function, i.e., the hat function $\phi_{i}(x), i=1,2, \ldots$, if $i \neq j$ and $i \neq j+1$. For $x_{j}$ and $x_{j+1}$, the associated piecewise linear basis functions $\phi_{j}(x)$ and $\phi_{j+1}(x)$ are modified. For example, $\phi_{j}(x)$ is defined as a piecewise linear function that satisfies

$$
\varphi_{j}\left(x_{j}\right)=1, \quad \varphi_{j}\left(x_{i}\right)=0, \quad\left[\varphi_{j}\right]=0, \quad\left[\beta \varphi_{j}^{\prime}\right]=0 .
$$

It has been derived by Li and Ito (2006) that

$$
\varphi_{j}(x)= \begin{cases}0, & 0 \leq x<x_{j-1}, \\ \frac{x-x_{j-1}}{h}, & x_{j-1} \leq x<x_{j}, \\ \frac{x_{j}-x}{D}+1, & x_{j} \leq x<\alpha \\ 0, & \alpha \leq x<x_{j+1}, \\ D & x_{j+1} \leq x \leq 1,\end{cases}
$$

where

$$
\rho=\frac{\beta_{1}}{\beta_{2}}, \quad D=h-\frac{\beta_{2}-\beta_{1}}{\beta_{2}}\left(x_{j+1}-\alpha\right),
$$

and

$$
\varphi_{j+1}(x)= \begin{cases}\frac{0,}{x-x_{j}} & 0 \leq x<x_{j}, \\ \frac{\rho\left(x-x_{j+1}\right)}{D}+1, & x_{j} \leq x<\alpha, \\ \frac{x_{j+2}-x}{h}, & x_{j+1} \leq x<x_{j+2}, \\ 0, & x_{j+2} \leq x \leq 1 .\end{cases}
$$

Let $V_{h,(0,1)} \triangleq \operatorname{Span}\left\{\varphi_{i}\right\}_{i=1}^{n-1}$ be the immersed finite element space for approximating $u$. We propose the following bilinear form for the problem (4): find $u_{h} \in$ $V_{h,(0,1)} \subset H_{0}^{1}(0,1)$ such that

$$
a\left(u_{h}, v_{h}\right)=\left(f, v_{h}\right), \quad \forall v_{h} \in V_{h,(0,1)} .
$$

In Fig. 2, we plot several regular finite element basis functions and two modified basis functions with different coefficients.

2.2. Error analysis for the 1D IFEM. Some error analysis of the 1D IFEM has been given by Li (1998) or $\mathrm{Li}$ and Ito (2006). Here we provide a somewhat different and more traditional analysis. As usual, we study the 

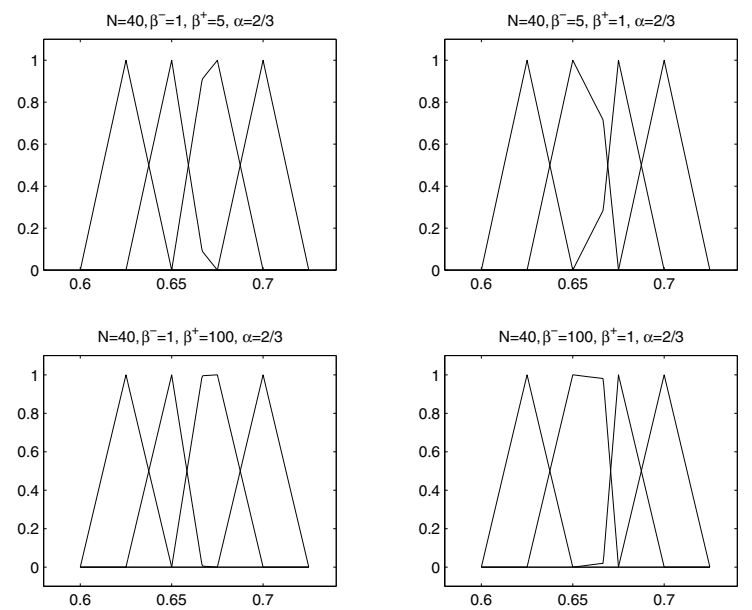

Fig. 2. Plots of some basis function near the interface with different $\beta^{-}=\beta_{1}$ and $\beta^{+}=\beta_{2}$. The interface is $\alpha=2 / 3$. Top left: $\beta^{-}=1, \beta^{+}=5$, top right: $\beta^{-}=5$, $\beta^{+}=1$, bottom left: $\beta^{-}=1, \beta^{+}=100$, bottom right: $\beta^{-}=100, \beta^{+}=1$.

approximation property of the IFE space $V_{h,(0,1)}$ so that we can bound the error of the finite element solution using that of the interpolation function.

Assuming that $x_{j} \leq \alpha<x_{j+1}$, we define the interpolation operator $\pi_{h}: H_{0}^{1}(0,1) \longrightarrow V_{h,(0,1)}$ as follows:

$$
\pi_{h} u(x)=\left\{\begin{array}{c}
\frac{x_{i+1}-x}{h} u\left(x_{i}\right)+\frac{x-x_{i}}{h} u\left(x_{i+1}\right), \\
x_{i}<x<x_{i+1}, \quad i \neq j, \\
\kappa\left(x-x_{j}\right)+u\left(x_{j}\right), \quad x_{j}<x \leq \alpha, \\
\kappa \frac{\beta_{1}}{\beta_{2}}\left(x-x_{j+1}\right)+u\left(x_{j+1}\right), \\
\alpha \leq x<x_{j+1},
\end{array}\right.
$$

where

$$
\kappa=\frac{\beta_{2}\left(u\left(x_{j+1}\right)-u\left(x_{j}\right)\right)}{\beta_{2}\left(\alpha-x_{j}\right)-\beta_{1}\left(\alpha-x_{j+1}\right)} .
$$

It is easy to check that $\pi_{h} u\left(x_{i}\right)=u\left(x_{i}\right), i=0, \ldots, n$, $\left[\pi_{h} u\right]=0$, and $\left[\beta \pi_{h} u^{\prime}\right]=0$.

Now we pay attention to the estimation of $\left\|u(x)-\pi_{h} u(x)\right\|_{0}$. Here, we define $E_{i}=$ $\left[x_{i}, x_{i+1}\right], i \neq j$ and $I=\left[x_{j}, x_{j+1}\right]$. For a regular element, we use the classical finite element method to estimate the error:

$$
\left\|u-\pi_{h} u\right\|_{1, E_{i}} \leq c h\|u\|_{2, E_{i}}
$$

We are going to focus on the error analysis for the element which contains the interface. We first define

$$
\begin{aligned}
& \widetilde{H}^{2}(0,1) \\
& \quad=\left\{v \in H_{0}^{1}(0,1), v \in H^{2}(0, \alpha), v \in H^{2}(\alpha, 1)\right\} .
\end{aligned}
$$

equipped with the norm and the semi-norm

$$
\|u\|_{\widetilde{H}^{2}(0,1)}^{2} \triangleq\|u\|_{H^{2}(0, \alpha)}^{2}+\|u\|_{H^{2}(\alpha, 1)}^{2}
$$

and

$$
|u|_{\widetilde{H}^{2}(0,1)}^{2} \triangleq|u|_{H^{2}(0, \alpha)}^{2}+|u|_{H^{2}(\alpha, 1)}^{2} .
$$

Then we have the following error estimate for the derivative approximation, $\kappa \sim u_{x}^{-}$from the left.

Lemma 1. If $u(x)$ is the solution of (4), then

$$
\begin{aligned}
& \left|u_{x}^{-}(\alpha)-\kappa\right| \leq c h^{\frac{1}{2}}\|u\|_{2, I}, \\
& \left|u_{x}^{+}(\alpha)-\kappa \rho\right| \leq \operatorname{ch}^{\frac{1}{2}}\|u\|_{2, I}
\end{aligned}
$$

Proof. Using the Taylor expansion at $\alpha$ and the jump conditions (i.e., (5)), we have

$$
\begin{aligned}
\left|u_{x}^{-}(\alpha)-\kappa\right| & \left|u_{x}^{-}(\alpha)-\frac{\beta_{2}\left(u\left(x_{j+1}\right)-u\left(x_{j}\right)\right)}{\beta_{2}\left(\alpha-x_{j}\right)-\beta_{1}\left(\alpha-x_{j+1}\right)}\right| \\
= & \mid u_{x}^{-}(\alpha)-\frac{\beta_{2}\left\{u^{+}(\alpha)+u_{x}^{+}(\alpha)\left(x_{j+1}-\alpha\right)\right\}}{\beta_{2}\left(\alpha-x_{j}\right)-\beta_{1}\left(\alpha-x_{j+1}\right)} \\
& -\beta_{2} \frac{\int_{\alpha}^{x_{j+1}} u^{\prime \prime}(t)\left(x_{j+1}-t\right) \mathrm{d} t}{\beta_{2}\left(\alpha-x_{j}\right)-\beta_{1}\left(\alpha-x_{j+1}\right)} \\
& -\beta_{2} \frac{u^{-}(\alpha)+u_{x}^{-}(\alpha)\left(x_{j}-\alpha\right)}{\beta_{2}\left(\alpha-x_{j}\right)-\beta_{1}\left(\alpha-x_{j+1}\right)} \\
& -\beta_{2} \frac{\int_{\alpha}^{x_{j}} u^{\prime \prime}(t)\left(x_{j}-t\right) \mathrm{d} t}{\beta_{2}\left(\alpha-x_{j}\right)-\beta_{1}\left(\alpha-x_{j+1}\right)} \mid \\
\leq & \left|\frac{\beta_{2} \int_{\alpha}^{x_{j+1}} u^{\prime \prime}(t)\left(x_{j+1}-t\right) \mathrm{d} t}{\beta_{2}\left(\alpha-x_{j}\right)-\beta_{1}\left(\alpha-x_{j+1}\right)}\right| \\
& +\left|\frac{\beta_{2} \int_{\alpha}^{x_{j}} u^{\prime \prime}(t)\left(x_{j}-t\right) \mathrm{d} t}{\beta_{2}\left(\alpha-x_{j}\right)-\beta_{1}\left(\alpha-x_{j+1}\right)}\right| \\
\leq & \frac{\beta_{\max }}{\beta_{\min }} \frac{h^{3 / 2}}{h}\|u\|_{2,\left(\alpha, x_{j+1}\right)}+\frac{\beta_{\max }}{\beta_{\min }} \frac{h^{3 / 2}}{h}\|u\|_{2,\left(x_{j}, \alpha\right)} \\
\leq & c h^{\frac{1}{2}}\|u\|_{2, I},
\end{aligned}
$$

where $c=2 \beta_{\max } / \beta_{\min }$ is a positive constant independent of the interface location, $\|u\|_{2, I}$ is the standard notation for $H^{2}$ norm of $u$ on an interval without the interface, and $\|u\|_{2, I}^{2}=\|u\|_{H^{2}\left(x_{j}, \alpha\right)}^{2}+\|u\|_{H^{2}\left(\alpha, x_{j+1}\right)}^{2}$ on the interface element $\left(x_{j}, x_{j+1}\right)$. This completes the proof of the lemma.

The lemma gives rough estimates of the first order derivative of the interpolation function from each side of 
the interface with an $O(\sqrt{h})$ convergence order compared with $O(h)$ in $H^{1}(0,1)$ of the interpolation function. Later on, we will explain our method to get a second order accurate derivative from each side of the interface.

In a similar way, we can prove that

$$
\left|u_{x}^{+}(\alpha)-\kappa \rho\right| \leq \operatorname{ch}^{\frac{1}{2}}\|u\|_{2, I} .
$$

2.3. Convergence analysis of the 1D IFEM. Although some error analysis is given by $\mathrm{Li}$ (1998), below we provide a different, more traditional finite element analysis with some results that are useful for accurate gradient computations at the interface. First we prove the following theorem on the accuracy of the interpolating function $\pi_{h} u$.

Theorem 1. If $u(x)$ is the solution of (4) and $\pi_{h} u(x)$ is the interpolation function defined in (8), then

$$
\left\|u-\pi_{h} u\right\|_{1, I} \leq \operatorname{ch}\|u\|_{2, I},
$$

where $c$ is a positive constant independent of the interface location.

Proof. If $x_{j} \leq x \leq \alpha$, then using the Taylor expansion we have

$$
\begin{aligned}
u(x)= & u\left(x_{j}\right)+u^{\prime}\left(x_{j}\right)\left(x-x_{j}\right) \\
& +\int_{x_{j}}^{x} u^{\prime \prime}(t)(x-t) \mathrm{d} t \\
= & u\left(x_{j}\right)+\left(u_{x}^{-}(\alpha)+\int_{\alpha}^{x_{j}} u^{\prime \prime}(x) d x\right)\left(x-x_{j}\right) \\
& +\int_{x_{j}}^{x} u^{\prime \prime}(t)(x-t) \mathrm{d} t \\
= & u\left(x_{j}\right)+u_{x}^{-}(\alpha)\left(x-x_{j}\right) \\
& +\left(\int_{\alpha}^{x_{j}} u^{\prime \prime}(x) d x\right)\left(x-x_{j}\right) \\
& +\int_{x_{j}}^{x} u^{\prime \prime}(t)(x-t) \mathrm{d} t .
\end{aligned}
$$

By (10) and the Cauchy-Schwartz inequality, we get

$$
\begin{aligned}
\mid u(x)- & \pi_{h} u(x) \mid \\
= & \mid\left(u_{x}^{-}(\alpha)-k\right)\left(x-x_{j}\right) \\
& +\left(\int_{\alpha}^{x_{j}} u^{\prime \prime}(x) \mathrm{d} x\right)\left(x-x_{j}\right) \mid \\
& +\left|\int_{x_{j}}^{x} u^{\prime \prime}(t)(x-t) \mathrm{d} t\right| \\
\leq & \operatorname{ch}^{\frac{3}{2}}\|u\|_{2, I},
\end{aligned}
$$

where $c$ is a generic constant (different from previous ones), and

$$
\begin{aligned}
& \left|\left(u(x)-\pi_{h} u(x)\right)^{\prime}\right| \\
& \quad=\left|u_{x}^{-}(\alpha)-k+\int_{\alpha}^{x_{j}} u^{\prime \prime}(x) d x+\int_{x_{j}}^{x} u^{\prime \prime}(t) \mathrm{d} t\right| \\
& \quad \leq \operatorname{ch}^{\frac{1}{2}}\|u\|_{2, I},
\end{aligned}
$$

where $\|u\|_{2, I}$ is the standard notation for $H^{2}$ norm of $u$ on an element.

If $\alpha \leq x \leq x_{j+1}$, the proof is similar. Thus, we also have

$$
\begin{gathered}
\left|u(x)-\pi_{h} u(x)\right| \leq \operatorname{ch}^{\frac{3}{2}}\|u\|_{2, I}, \\
\left|\left(u(x)-\pi_{h} u(x)\right)^{\prime}\right| \leq c h^{\frac{1}{2}}\|u\|_{2, I} .
\end{gathered}
$$
follows:

We proceed with the remainder of the proof as

$$
\begin{aligned}
\| u(x) & -\pi_{h} u(x) \|_{0, I} \\
= & \left(\int_{x_{j}}^{\alpha}\left(u(x)-\pi_{h} u(x)\right)^{2} \mathrm{~d} x\right. \\
& \left.+\int_{\alpha}^{x_{j+1}}\left(u(x)-\pi_{h} u(x)\right)^{2} \mathrm{~d} x\right)^{\frac{1}{2}} \\
\leq & c\left(\int_{x_{j}}^{\alpha} h^{3}\|u\|_{2, I}^{2} d x+\int_{\alpha}^{x_{j+1}} h^{3}\|u\|_{2, I}^{2} \mathrm{~d} x\right)^{\frac{1}{2}} \\
\leq & c h^{3 / 2}\|u\|_{2, I}^{2}\left(\int_{x_{j}}^{\alpha} \mathrm{d} x+\int_{\alpha}^{x_{j+1}} \mathrm{~d} x\right)^{\frac{1}{2}} \\
\leq & c h^{2}\|u\|_{2, I},
\end{aligned}
$$

in $L^{2}$, and we continue to $H^{1}$,

$$
\begin{aligned}
\mid u(x) & -\left.\pi_{h} u(x)\right|_{1, I} \\
= & \left(\int_{x_{j}}^{\alpha}\left(\left(u(x)-\pi_{h} u(x)\right)^{\prime}\right)^{2} \mathrm{~d} x\right. \\
& \left.+\int_{\alpha}^{x_{j+1}}\left(\left(u(x)-\pi_{h} u(x)\right)^{\prime}\right)^{2} \mathrm{~d} x\right)^{\frac{1}{2}} \\
\leq & c\left(\int_{x_{j}}^{\alpha} h\|u\|_{2, I}^{2} \mathrm{~d} x+\int_{\alpha}^{x_{j+1}} h\|u\|_{2, I}^{2} \mathrm{~d} x\right)^{\frac{1}{2}} \\
\leq & c h\|u\|_{2, I} .
\end{aligned}
$$

Combining all of the above, we get

$$
\begin{aligned}
\left\|u-\pi_{h} u\right\|_{1, I} & =\left(\left\|u-\pi_{h} u\right\|_{0, I}^{2}+\left|u-\pi_{h} u\right|_{1, I}^{2}\right)^{\frac{1}{2}} \\
& \leq \operatorname{ch}\|u\|_{2, I},
\end{aligned}
$$

which completes the proof.

The following theorem states that the IFEM in 1D provides optimal convergence like the FEM for regular problems. 
Theorem 2. If $u(x)$ is the solution of (4), and $\pi_{h} u(x)$ is the interpolating function defined in (8), then

$$
\left\|u-\pi_{h} u\right\|_{1} \leq c h\|u\|_{2},
$$

Proof. We use (9) and (11) to get

$$
\begin{aligned}
\| u- & \pi_{h} u \|_{1} \\
= & \left(\int_{0}^{1}\left(u(x)-\pi_{h} u(x)\right)^{2} \mathrm{~d} x\right. \\
& \left.+\int_{0}^{1}\left(\left(u(x)-\pi_{h} u(x)\right)^{\prime}\right)^{2} \mathrm{~d} x\right)^{\frac{1}{2}} \\
= & \left(\sum_{E_{i}}\left\|u-\pi_{h} u\right\|_{1, E_{i}}^{2}+\left\|u-\pi_{h} u\right\|_{1, I}^{2}\right)^{\frac{1}{2}} \\
\leq & \left(\sum_{E_{i}} c h^{2}\|u\|_{2, E_{i}}^{2}+c h^{2}\|u\|_{2, I}^{2}\right)^{\frac{1}{2}} \\
\leq & \operatorname{ch}\left(\sum_{E_{i}}\|u\|_{2, E_{i}}^{2}+\|u\|_{2, I}^{2}\right)^{\frac{1}{2}} \\
\leq & \operatorname{ch}\|u\|_{2} \cdot
\end{aligned}
$$

This completes the proof of the theorem.

2.4. Accurate flux computation at the left of the interface. In this sub-section, we explain how to get an accurate flux or the first order derivative of the solution at the interface from the left side of the interface. The method is based on the approach proposed by Wheeler (1974) for flux computations at boundaries. It uses the Galerkin solution of the problem and it is different from other posterior error analysis.

We define

$$
\Gamma_{\alpha}^{-} \triangleq \frac{1}{\alpha}\left\{\left(\beta u_{h}^{\prime}, 1\right)_{(0, \alpha)}+\left(q u_{h}-f, x\right)_{(0, \alpha)}\right\}
$$

as an approximation to the exact flux $\beta_{1} u_{x}^{-}(\alpha)$. Below we show that this is a second order approximation, which improves the accuracy of the flux by one order compared with the estimate in (12).

Theorem 3. If $u(x)$ is the solution of (4), $u_{h}(x)$ is the Galerkin approximation of the solution of $u(x), \Gamma_{\alpha}^{-}$is as defined above, then

$$
\left|\Gamma_{\alpha}^{-}-\beta_{1} u_{x}^{-}(\alpha)\right| \leq c h^{2}\|u\|_{2} .
$$

Proof. We define $Y \in V_{h}$ as a function that satisfies $Y(0)=0$ and

$$
\begin{array}{r}
\left(\beta Y^{\prime}, v_{h}^{\prime}\right)_{(0, \alpha)}+\left(q Y-f, v_{h}\right)_{(0, \alpha)}=\beta_{1} u_{x}^{-}(\alpha) v_{h}(\alpha), \\
\forall v_{h} \in V_{h}, \text { and } v_{h}(0)=0 .
\end{array}
$$

Subtracting (13) with $v_{h}=x / \alpha$ from (15), we have

$$
\begin{aligned}
& \left|\Gamma_{\alpha}^{-}-\beta_{1} u_{x}^{-}(\alpha)\right| \\
& \quad=\frac{1}{\alpha}\left|\left(\beta\left(u_{h}-Y\right)^{\prime}, 1\right)_{(0, \alpha)}+\left(q\left(u_{h}-Y\right), x\right)_{(0, \alpha)}\right| \\
& \quad \leq c\left\{\left\|u_{h}-Y\right\|_{0}+\left|\left(u_{h}-Y\right)(\alpha)\right|\right\} .
\end{aligned}
$$

From (7) and (15) we can see that

$$
\begin{array}{r}
\left(\beta\left(u_{h}-Y\right)^{\prime}, v_{h}^{\prime}\right)_{(0, \alpha)}+\left(q\left(u_{h}-Y\right), v_{h}\right)_{(0, \alpha)}=0, \\
\forall v_{h} \in V_{h,(0, \alpha)} .
\end{array}
$$

Set $w=u_{h}-Y$ and $v_{h}=w-x w(\alpha)$; we then get the following equation:

$$
\begin{aligned}
& \left(\beta w^{\prime}, w^{\prime}\right)_{(0, \alpha)}+(q w, w)_{(0, \alpha)} \\
& \quad=\left(\beta w^{\prime}, w(\alpha)\right)_{(0, \alpha)}+(q w, x w(\alpha))_{(0, \alpha)},
\end{aligned}
$$

and thus we have

$$
\|w\|_{1} \leq c\left\{\|w\|_{0}+|w(\alpha)|\right\} .
$$

Next we construct the following auxiliary problem. Let $\varphi \in H^{2}(0, \alpha) \cap \widetilde{H}^{1}(0, \alpha)$ be the solution of

$$
\left\{\begin{array}{l}
L^{*} \varphi=-w, \quad w \in(0, \alpha), \\
\varphi(0)=\varphi(\alpha)=0
\end{array}\right.
$$

where $L^{*} \varphi=-\left(\beta \varphi^{\prime}\right)^{\prime}+q \varphi$. We also assume that

$$
\|\varphi\|_{2} \leq c\|w\|_{0} .
$$

Then, for an appropriately chosen $\pi \varphi \in V_{h,(0, \alpha)}$, we proceed to get the following:

$$
\begin{aligned}
(w, w)_{(0, \alpha)}= & \left|-\left(w, L^{*} \varphi\right)_{(0, \alpha)}\right| \\
= & \left|\left(w,\left(\beta \varphi^{\prime}\right)^{\prime}-q \varphi\right)_{(0, \alpha)}\right| \\
\leq & \left|\left(\beta w^{\prime}, \varphi^{\prime}-(\pi \varphi)^{\prime}\right)_{(0, \alpha)}\right| \\
& +\left|(q w, \varphi-x)_{(0, \alpha)}\right|+\left|\left(\beta \varphi^{\prime} w\right)(\alpha)\right| \\
\leq & c\left\{\|w\|_{1}\|\varphi-\pi \varphi\|_{1}+\left|\varphi^{\prime}(\alpha)\right||w(\alpha)|\right\} \\
\leq & c\left\{h\|w\|_{1}\|\varphi\|_{2}+\|w\|_{0}|w(\alpha)|\right\} \\
\leq & c\|w\|_{0}\left\{h\|w\|_{1}+|w(\alpha)|\right\} .
\end{aligned}
$$

The above yields

$$
\|w\|_{0} \leq c\left\{h\|w\|_{1}+|w(\alpha)|\right\} .
$$

For $h$ sufficiently small, (16) and (17) imply that

$$
\|w\|_{0} \leq c|w(\alpha)|
$$

where $c$ is a positive constant independent of the interface $\alpha$. 
We now derive an estimate of $|w(\alpha)|$, using the new auxiliary problem

$$
\left\{\begin{array}{l}
L^{*} \xi=0, \quad \xi \in(0, \alpha) \\
\xi(0)=0, \quad \beta_{1}(\alpha) \xi^{\prime}(\alpha)=1
\end{array}\right.
$$

Let $\eta=u-Y$; then we can get

$$
\begin{aligned}
\left(\beta \eta^{\prime}, v_{h}\right)_{(0, \alpha)}+ & \left(q \eta, v_{h}\right)_{(0, \alpha)}=0 \\
& \forall v_{h} \in V_{h} \text { such that } v_{h}(0)=0 .
\end{aligned}
$$

Furthermore, using

$$
\begin{aligned}
0 & =-\left(\eta, L^{*} \xi\right)_{(0, \alpha)} \\
& =-\left(\eta,-\left(\beta \xi^{\prime}\right)^{\prime}+q \xi\right)_{(0, \alpha)} \\
& =\left(\eta,\left(\beta \xi^{\prime}\right)^{\prime}-q \xi\right)_{(0, \alpha)} \\
& =\left(-\beta \eta^{\prime}, \xi^{\prime}\right)_{(0, \alpha)}+(-q \eta, \xi)_{(0, \alpha)}+\eta(\alpha),
\end{aligned}
$$

we have

$$
\begin{aligned}
|\eta(\alpha)| & =\left|\left(\beta \eta^{\prime}, \xi^{\prime}\right)_{(0, \alpha)}+(q \eta, \xi)_{(0, \alpha)}\right| \\
& \leq c\|\eta\|_{1}\|\xi-x\|_{1} \\
& \leq c h\|\eta\|_{1} \\
& \leq c h^{2}\|u\|_{2} .
\end{aligned}
$$

Finally, we get

$$
\begin{aligned}
\left|\Gamma_{\alpha}^{-}-\beta_{1} u_{x}^{-}(\alpha)\right| & \leq c\left|\left(u_{h}-Y\right)(\alpha)\right| \\
& \leq c\left\{\left|\left(u-u_{h}\right)(\alpha)\right|+|(u-Y)(\alpha)|\right\} \\
& \leq c h^{2}\|u\|_{2} .
\end{aligned}
$$

This completes the proof of the theorem.

Approximation of the flux from the right side of the interface. In a similar way, we can get the second order accurate flux, $-\beta^{+} u_{x}^{+}$, from the right side of the interface

$$
\Gamma_{\alpha}^{+} \triangleq \frac{1}{1-\alpha}\left\{\left(\beta u_{h}^{\prime},-1\right)_{(\alpha, 1)}+\left(q u_{h}-f, 1-x\right)_{(\alpha, 1)}\right\} .
$$

We also have the following error bound:

$$
\left|\Gamma_{\alpha}^{+}+\beta^{+} u_{x}^{+}(\alpha)\right| \leq c h^{2}\|u\|_{2} .
$$

Approximation of fluxes at the boundary. The approach for accurate flux computations at the interface can be applied to flux computation from the left and right boundaries as expressed below. We define approximations $\Gamma_{0}$ and $\Gamma_{1}$ to the fluxes, $\beta_{1} u^{\prime}(0)$ and $\beta_{2} u^{\prime}(1)$, respectively:

$$
\Gamma_{0} \triangleq\left(\beta u_{h}^{\prime},-1\right)+\left(q u_{h}-f, 1-x\right),
$$

$$
\Gamma_{1} \triangleq\left(\beta u_{h}^{\prime}, 1\right)+\left(q u_{h}-f, x\right) .
$$

Then $\Gamma_{0}$ and $\Gamma_{1}$ are second order approximations to the flux from the left and right boundaries as stated in the following theorem. We skip the proof since it is similar to that for the flux from the left side of the interface.

Theorem 4. If $u(x)$ is the solution of (4), $u_{h}(x)$ is the Galerkin approximation of the solution of $u(x)$ while $\Gamma_{0}$ and $\Gamma_{1}$ are as defined above, then

$$
\left|\Gamma_{0}+\beta_{1} u^{\prime}(0)\right|+\left|\Gamma_{1}-\beta_{2} u^{\prime}(1)\right| \leq c h^{2}\|u\|_{2} .
$$

2.5. Numerical experiments in 1D. We present one example below that is taken from the work of $\mathrm{Li}$ and Ito (2006). The exact solution is

$$
u(x)= \begin{cases}x^{4} / \beta^{-}, & \text {if } 0<x<\alpha, \\ x^{4} / \beta^{+}+\left(1 / \beta^{-}-1 / \beta^{+}\right) \alpha^{4}, & \text { if } \alpha<x<1,\end{cases}
$$

where $0<\alpha<1$ is an interface. The solution satisfies the ODE $-\left(\beta u^{\prime}\right)^{\prime}=f(x)$, where $f(x)=-12 x^{2}$. In this example, $q(x)=0$ and $[u]=0$ and $\left[\beta u^{\prime}\right]=0$, but $\left[u^{\prime}\right] \neq 0$.

In Table 1, we show a grid refinement analysis for the proposed method with $\alpha=1 / 3, \beta^{-}=2, \beta^{+}=10$. Thus, the interface $\alpha$ is not a nodal point. We measure the error for the solution $u(x)$ in the entire domain $(0,1)$ in the second column using the strongest $L^{\infty}$ norm. We estimate the convergence order using $p=\log \left(E_{n} / E_{2 N}\right) / \log 2$ in the third column. As usual, since the relative location between the underlying grid and the interface $\alpha$ is not fixed, the convergence order fluctuates. The average convergence order is 1.983 . In the third column, we list the grid refinement analysis for

$$
u_{x}^{-}=\lim _{x \rightarrow \alpha, x<\alpha} u^{\prime}(x),
$$

that is, the first order derivative from the left side of the interface, and we observe clear second order convergence as shown in the fifth column.

Table 1. Grid refinement analysis of the proposed method with $\alpha=1 / 3, \beta^{-}=2, \beta^{+}=10$. The second column is the $L^{\infty}$ error of the solution in the entire domain $(0,1)$.

\begin{tabular}{|c|c|c|c|c|}
\hline $\mathrm{N}$ & $\left\|u-u_{h}\right\|_{L^{\infty}}$ & Order & $E_{u_{x}}$ & Order \\
\hline \hline 16 & $3.395 \mathrm{E}-05$ & & $3.870 \mathrm{E}-03$ & \\
\hline 32 & $1.547 \mathrm{E}-05$ & 1.134 & $7.980 \mathrm{E}-04$ & 2.278 \\
\hline 64 & $2.191 \mathrm{E}-06$ & 2.820 & $1.562 \mathrm{E}-04$ & 2.353 \\
\hline 128 & $9.732 \mathrm{E}-07$ & 1.171 & $3.892 \mathrm{E}-05$ & 2.005 \\
\hline 256 & $1.413 \mathrm{E}-07$ & 2.784 & $8.475 \mathrm{E}-06$ & 2.199 \\
\hline 512 & $6.088 \mathrm{E}-08$ & 1.215 & $2.263 \mathrm{E}-06$ & 1.905 \\
\hline 1024 & $8.900 \mathrm{E}-09$ & 2.774 & $5.098 \mathrm{E}-07$ & 2.150 \\
\hline
\end{tabular}

The fourth column is the error in the first order derivative $u_{x}^{-}$, that is, from the left side of the interface, 
defined as $E_{u_{x}}=\max \left|u^{\prime}\left(\alpha^{ \pm}\right)-u_{h}^{\prime}\left(\alpha^{ \pm}\right)\right|$. The average convergence order for the solution and $u_{x}^{-}$is 1.983 and 2.148 , respectively.

\section{Numerical method and experiments for the 2D interface problem}

The results in the previous section are optimal since both the solution and the flux (at the interface or boundaries) use piecewise linear finite element space. However, it is still an open question how to apply the approach to 2D problems with a curved interface. In this section, we provide an alternative approach that is similar to a mixed finite element method but only in a small tube around the interface.

In this section, the elliptic interface problem is

$$
\begin{aligned}
-\nabla \cdot(\beta \nabla u(\mathbf{x}))+q(\mathbf{x}) u(\mathbf{x}) & =f(\mathbf{x}), \\
\mathbf{x} & \in \Omega_{i}, \quad i=1,2,
\end{aligned}
$$

where $q(\mathbf{x}) \in L^{\infty}(\Omega) \geq 0, \Omega=\Omega^{-} \cup \Omega^{+}, \beta(\mathbf{x})$ is a piecewise positive constant as in (2) and has a finite jump discontinuity across a closed interface $\Gamma \in C^{1}$ in the solution domain.

In our new method, we introduce a tube that contains the interface with a diameter $2 \epsilon$ as

$$
\Omega_{\epsilon}=\left\{\mathbf{x} \in \Omega_{1} \cup \Omega_{2}, \quad d(\mathbf{x}, \Gamma) \leq \epsilon\right\},
$$

where $d(\mathbf{x}, \Gamma)$ is the distance between $\mathbf{x}$ and the interface $\Gamma$. In the tube $\Omega_{\epsilon}$, we introduce the flux as a separate variable vector $\mathbf{v}$ that can be considered an augmented variable. Thus, in addition to the PDE (20) in the entire domain, we also have the following equations:

$$
\begin{gathered}
-\beta \nabla u=\mathbf{v}, \\
\nabla \cdot \mathbf{v}+q u=f, \quad
\end{gathered}
$$

Next we define the following functional spaces:

$$
\begin{aligned}
H_{0}^{1} & =\left\{\phi \in H^{1}(\Omega), \phi=0 \text { on } \partial \Omega\right\}, \\
W & =\left\{w \in L^{2}\left(\Omega_{\epsilon}\right)\right\}, \\
L_{g} & =\left\{\mathbf{g} \in\left(L^{2}\left(\Omega_{\epsilon}\right)\right)^{2}\right\},
\end{aligned}
$$

assuming a homogenous boundary condition along $\partial \Omega_{2}$.

We can easily get the following weak form for $u$ (in the entire domain) and $\mathbf{g}$ (in $\Omega_{\epsilon}$ ):

$$
\begin{gathered}
\left(\beta_{i} \nabla u, \nabla \phi\right)+(q u, \phi)=(f, \phi) \text { in } \Omega_{i}, i=1,2, \\
-\left(\beta_{i} \nabla u, \mathbf{g}\right)=(\mathbf{v}, \mathbf{g}) \text { in } \Omega_{\epsilon} \cap \Omega_{i}, \quad i=1,2, \\
(\nabla \cdot \mathbf{v}, w)+(q u, w)=(f, w) \quad \text { in } \Omega_{\epsilon},
\end{gathered}
$$

where the inner product is in the regular $L^{2}$ sense and the quantities $\phi, \mathbf{g}$, and $w$ are from the spaces defined above.

There are two directions of intuitive reasoning behind the new algorithm. We know that the mixed formulation would improve the gradient computation. If we are only interested in the gradient from each side of the interface, then we just need to use a small tube for the computation. The second consideration is that if we set the flux $\mathbf{v}=$ $\beta \nabla u$ along the interface as an unknown in addition to the solution $u$, and then discretize the whole system with high order discretization (second order in the manuscript), then we would expect the error for the unknown flux $\mathbf{v}$ to have the same order of accuracy as for the discretization. In discretization, we use piecewise linear functions for $\phi$ and $\mathbf{g}$ as usual, and piecewise constant functions for $w$. The new augmented method enlarged the system by (22) and (23). In terms of the stiffness matrix, an additional $n_{v}$ number of columns are added to the stiffness matrix, where $n_{v}$ is the number of extra unknowns $\mathbf{v}$ in the tube $\Omega_{\epsilon}$. As a result, the stiffness matrix becomes rectangular instead of square. We used singular value decomposition (SVD) to solve the resultant rectangular system. Since $\mathbf{v}$ has co-dimension one compared with that of $u$, the additional extra cost is negligible compared with that of the elliptic solver on the entire domain.

3.1. Numerical experiments in 2D. Let $\Omega_{2}$ be a unit circle centered at $(0,0)$ with radius $R=1$. In our numerical test, we take $q=0$. Let $\Gamma$ be an interface inside the unit circle with radius $R=0.9$. The tube width is taken as $\epsilon=3 h$, that is, three layers from each side of the interface. The exact solution is

$$
u(x, y)=\sin x \cos y
$$

in the entire domain so that the solution is continuous, but the flux $\beta \nabla u$ is discontinuous for the test problem. The coefficient is taken as $\beta_{1}=100$ and $\beta_{2}=1$. The source terms and the boundary condition are determined accordingly. In Table 2, the $L^{2}$ norm errors of $u, \mathbf{v}$ and the $H^{1}$ norm error of $u$ are reported. $L^{2}(\Omega), H^{1}(\Omega)$ are used for the solution $u$, that is, in the entire domain, while $L^{2}(\Gamma)$ is used for the flux $\mathbf{v}$ along the interface. The first column $N$ is the number of mesh lines in the coordinate directions. The results indicate that the new augmented method worked as expected. The convergence rate is shown in Table 3.

In Table 3, a comparison of the convergence order between the standard finite element method and the new augmented approach is presented. We observe that the new approach provides much better accuracy for the flux (gradient). Now we have super-convergence for the gradient. Super-convergence here is the result of the convergence that is faster than the original method. For the finite element method with the piecewise linear function space, it is well known that the flux is first order accurate in the $L^{2}$ norm. In our manuscript, we proposed a method to reconstruct the flux at the interface and showed that the reconstructed flux has second order convergence 
Table 2. Grid refinement analysis of the proposed method. The $H^{1}$ norm of $u$ is the same as the $L^{2}$ error of $\mathbf{v}$ because the error in the gradient is dominated compared with that of $u$.

\begin{tabular}{|c|c|c|c|}
\hline$N$ & $L^{2}$ error of $u$ & $H^{1}$ error of $u$ & $L^{2}$ error of $\mathbf{v}$ \\
\hline \hline 8 & $9.96 \mathrm{e}-3$ & $5.67 \mathrm{e}-1$ & $5.67 \mathrm{e}-1$ \\
\hline 16 & $2.48 \mathrm{e}-3$ & $1.75 \mathrm{e}-1$ & $1.75 \mathrm{e}-1$ \\
\hline 32 & $7.77 \mathrm{e}-4$ & $4.14 \mathrm{e}-2$ & $4.14 \mathrm{e}-2$ \\
\hline 64 & $1.81 \mathrm{e}-4$ & $1.04 \mathrm{e}-2$ & $1.04 \mathrm{e}-2$ \\
\hline 128 & $4.71 \mathrm{e}-5$ & $5.95 \mathrm{e}-3$ & $5.95 \mathrm{e}-3$ \\
\hline 256 & $1.15 \mathrm{e}-5$ & $1.54 \mathrm{e}-3$ & $1.54 \mathrm{e}-3$ \\
\hline 512 & $2.92 \mathrm{e}-6$ & $3.80 \mathrm{e}-4$ & $3.80 \mathrm{e}-4$ \\
\hline
\end{tabular}

in 1D. For 2D problems, the computed fluxes at the interface have been observed with super-convergence with order $1.5 \sim 1.7$.

Table 3. Comparison of the convergence order between the standard FEM and the new augmented approach.

\begin{tabular}{|c|c|c|c|}
\hline Quantity & $u$ & $u$ & $\mathbf{v}$ \\
\hline \hline Norm & $L^{2}$ & $H^{\perp}$ & $L^{2}$ \\
\hline Order (standard FEM) & 1.98 & 1.03 & 1.03 \\
\hline Order (new method) & 1.94 & 1.72 & 1.72 \\
\hline
\end{tabular}

Next, in Table 4 we present the results with different interface locations including the case that covers the entire domain $\left(r_{i}=0\right)$ so that we get the gradient in the entire domain as well, where $r_{i}$ is the radius of the interface. Of course, the computational cost also increased. As we expected, we have second order convergence in the $L^{2}$ norm for the solution, and roughly a 1.70 order for the flux (gradient). In this case, the accuracy of the gradient is improved by about 70 percent.

Table 4. Comparison of the convergence order for various locations of the interface, where $r_{i}$ is the radius of the interface.

\begin{tabular}{|c|c|c|}
\hline$r_{i}$ & Order in $L^{2}$ of $u$ & Order in $H^{1}$ \\
\hline \hline 0.9 & 1.94 & 1.72 \\
\hline 0.99 & 1.94 & 1.70 \\
\hline 0 & 1.93 & 1.70 \\
\hline
\end{tabular}

Now we show the result with a non-zero $q(x, y)$. We use the same solution above with $q(x, y)=1$. The source term is modified accordingly. In Table 5, we show the grid refinement analysis of the error in $L^{2}$ and $H^{1}$ norm. We observe the same behavior with similar convergence orders. The average convergence orders are 1.92 for the $L^{2}$ norm and 1.71 for the $H^{1}$ norm. Note that, the $L^{2}$ norm of the error in $\mathbf{v}$ is the same as the $H^{1}$ norm, as explained earlier.
Table 5. Grid refinement analysis of the proposed method when $q(x, y)=1$. The average convergence orders are 1.92 for the $L^{2}$ norm and 1.71 for the $H^{1}$ norm.

\begin{tabular}{|c|c|c|}
\hline$N$ & $L^{2}$ error of $u$ & $H^{1}$ error of $u$ \\
\hline \hline 8 & $9.96 \mathrm{e}-3$ & $5.67 \mathrm{e}-1$ \\
\hline 16 & $3.51 \mathrm{e}-3$ & $2.61 \mathrm{e}-1$ \\
\hline 32 & $1.17 \mathrm{e}-4$ & $6.54 \mathrm{e}-2$ \\
\hline 64 & $2.81 \mathrm{e}-4$ & $1.66 \mathrm{e}-2$ \\
\hline 128 & $7.24 \mathrm{e}-5$ & $9.13 \mathrm{e}-3$ \\
\hline 256 & $1.85 \mathrm{e}-5$ & $2.31 \mathrm{e}-3$ \\
\hline 512 & $4.62 \mathrm{e}-6$ & $5.79 \mathrm{e}-4$ \\
\hline
\end{tabular}

In the previous example, the solution is the same in the entire domain although the flux is discontinuous. Below we present another example in which the solution is different in a different domain. The outer boundary is $R=2$ :

$$
u(x, y)= \begin{cases}\left(x^{2}+y^{2}\right)^{2} & \text { if } r>1 \\ \left(x^{2}+y^{2}\right) & \text { if } r \leq 1\end{cases}
$$

where $r=\sqrt{x^{2}+y^{2}}$. The source term $f(x, y)$, and the Dirichlet boundary condition are determined from the true solution. In this example, the solution is continuous, that is, $[u]=0$, but the flux jump is non-homogenous.

We tested our new method with large jump ratios, $\beta_{2}: \beta_{1}=1000: 1$ and $\beta_{2}: \beta_{1}=1: 1000$. In Table 6, we present the results with different widths of the tube including the case that covers the entire domain so that we get the gradient in the entire domain as well. As we expected, we have second order convergence in the $L^{2}$ norm for the solution, and roughly a 1.54 order for the flux (gradient), as in the thin tube case. Compared with the standard finite element method, the accuracy of the computed gradient is improved by more than 50 percent. Note that the results are almost the same as for the new gradient recovery technique using a posterior approach (Guo and Yang, 2017), in which the rate of the recovered gradient is around 1.5. Note that, the convergence order for the gradient is about 1.54, which is lower than the previous case, possibly due to the non-homogenous flux jump.

Table 6. Comparison of the convergence order for various widths of the tube.

\begin{tabular}{|c|c|c|}
\hline Width $(\epsilon)$ & Order in $L^{2}$ of $u$ & Order in $H^{1}$ \\
\hline \hline $3 h$ & 1.96 & 1.53 \\
\hline $10 h$ & 1.96 & 1.56 \\
\hline 2 (entire domain) & 1.96 & 1.56 \\
\hline
\end{tabular}

\section{Conclusions}

In this paper, we discussed two methods to enhance the accuracy of the computed flux at the interface for the 
elliptic interface problem. One is for one-dimensional problems in which we use a simple weak form to get second order accurate fluxes at the interface from each side. We also have a rigorous analysis for the approach. The other one is an augmented method for two dimensional interface problems. Numerical examples show that we get better than super-convergence (about a $1.50 \sim 1.70$ order) for the computed fluxes at the interface from each side. For the two dimensional algorithm, the theoretical analysis is still an open challenge.

\section{Acknowledgment}

We would like to thank the referees for many valuable suggestions and comments. F. Qin is partially supported by the China NSF grants 11671209, 11671210 and the NSF of Jiangsu Province, China (grant no. BK20160880). Z. Ma is supported by the China National Special Fund: 2012DFA60830, and Z. Li is partially supported by the US NSF grant DMS-1522768 as well as the China NSF grants 11371199 and 11471166 .

\section{References}

Adams, R. and Fournier, J. (2003). Sobolev Spaces. Second Edition, Academic Press, Cambridge, MA.

An, N. and Chen, H. (2014). A partially penalty immersed interface finite element method for anisotropic elliptic interface problems, Numerical Methods for Partial Differential Equations 30(6): 1984-2028.

Anitescu, C. (2017). Open source 3D Matlab isogeometric analysis code, https://sourceforge.net/u/ cmechanicsos/profile/

Babuška, I. (1970). The finite element method for elliptic equations with discontinuous coefficients, Computing 5(3): 207-213.

Bramble, J. and King, J. (1996). A finite element method for interface problems in domains with smooth boundaries and interfaces, Advances in Computational Mathematics 6(1): 109-138.

Brenner, S. and Scott, R. (2007). The Mathematical Theory of Finite Element Methods, Springer, New York, NY.

Cao, W., Zhang, X. and Zhang, Z. (2017). Superconvergence of immersed finite element methods for interface problems, Advances in Computational Mathematics 43(4): 795-821.

Carstensen, C., Gallistl, D., Hellwing, F. and Weggler, L. (2014). Low-order DPG-FEM for an elliptic PDE, Computers \& Mathematics with Applications 68(11): 1503-1512.

Chen, Z. and Zou, J. (1998). Finite element methods and their convergence for elliptic and parabolic interface problems, Numerische Mathematik 79(2): 175-202.

Chou, S. (2012). An immersed linear finite element method with interface flux capturing recovery, Discrete and Continuous Dynamical Systems B 17(7): 2343-2357.
Chou, S.H., Kwak, D.Y. and Wee, K. (2010). Optimal convergence analysis of an immersed interface finite element method, Advances in Computational Mathematics 33(2): 149-168.

Douglas Jr, J., Dupont, T. and Wheeler, M. (1974). A Galerkin procedure for approximating the flux on the boundary for elliptic and parabolic boundary value problems, $R e$ vue française d'automatique, informatique, recherche opérationnelle. Analyse numérique 8(2): 47-59.

Guo, H. and Yang, X. (2017). Gradient recovery for elliptic interface problem. II: Immersed finite element methods, Journal of Computational Physics 338: 606-619.

He, X., Lin, T. and Lin, Y. (2011). Immersed finite element methods for elliptic interface problems with non-homogeneous jump conditions, International Journal of Numerical Analysis and Modeling 8(2): 284-301.

Ji, H., Chen, J. and Li, Z. (2016). A new augmented immersed finite element method without using SVD interpolations, Numerical Algorithms 71(2): 395-416.

Karczewska, A., Rozmej P., Szczeciński, M. and Boguniewicz, B. (2016). A finite element method for extended $\mathrm{KdV}$ equations, International Journal of Applied Mathematics and Computer Science 26(3): 555-567, DOI: 10.1515/amcs-2016-0039.

Kevorkian, J. (1990). Partial Differential Equations: Analytical Solution, Springer, New York, NY.

Kwak, D.Y., Wee, K. and Chang, K. (2010). An analysis of a broken $p_{1}$ nonconforming finite element method for interface problems, SIAM Journal on Numerical Analysis 48(6): 2117-2134.

Li, Z. (1998). The immersed interface method using a finite element formulation, Applied Numerical Mathematics 27(3): 253-267.

Li, Z. and Ito, K. (2006). The Immersed Interface Method: Numerical Solutions of PDEs Involving Interfaces and Irregular Domains, SIAM, Philadelphia, PA.

Li, Z., Lin, T. and Wu, X. (2003). New Cartesian grid methods for interface problems using the finite element formulation, Numerische Mathematik 96(1): 61-98.

Lin, T., Lin, Y. and Zhang, X. (2015). Partially penalized immersed finite element methods for elliptic interface problems, SIAM Journal on Numerical Analysis 53(2): 1121-1144.

Lin, T. and Zhang, X. (2012). Linear and bilinear immersed finite elements for planar elasticity interface problems, Journal of Computational and Applied Mathematics 236(18): 4681-4699.

Sutton, A. and Balluffi, R. (1995). Interfaces in Crystalline Materials, Clarendon Press, Oxford.

Tartar, L. (2007). An Introduction to Sobolev Spaces and Interpolation Spaces, Springer, New York, NY.

Wahlbin, L. (1995). Superconvergence in Galerkin Finite Element Methods, Springer, New York, NY.

Wheeler, M. (1974). A Galerkin procedure for estimating the flux for two-point boundary value problems, SIAM Journal on Numerical Analysis 11(4): 764-768. 
Xu, J. (1982). Error estimates of the finite element method for the 2nd order elliptic equations with discontinuous coefficients, Journal of Xiangtan University 1(1): 1-5.

Yang, X., Li, B. and Li, Z. (2002). The immersed interface method for elasticity problems with interfaces, Dynamics of Continuous, Discrete and Impulsive Systems 10(5): 783-808.

Zhang, Z. and Naga, A. (2005). A new finite element gradient recovery method: Superconvergence property, SIAM Journal on Scientific Computing 26(4): 1192-1213.

Zienkiewicz, O. and Taylor, R. (2000). The Finite Element Method: Solid Mechanics, Butterworth-Heinemann, Oxford.

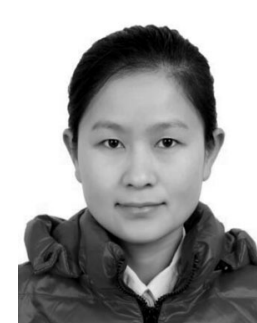

Fangfang Qin received her $\mathrm{PhD}$ degree in the School of Mathematical Sciences from Nanjing Normal University, Nanjing, China, in 2017. She was a visiting scholar in the Department of Mathematics at North Carolina State University from May 2016 to May 2017. Her research interests are numerical analysis and scientific computing. In particular, she works on numerical methods for partial differential equations and applications. Presently, her research focuses on immersed finite element methods on interface problems.
Zhaohui Wang received his PhD degree from North Carolina State University, USA, in 2016. Now he is working in industry.

Zhijie Ma is a professor of water resource and hydropower, China Institute of Water Resource and Hydropower Research (IWHR). He earned his $\mathrm{PhD}$ degree at the Wuhan University of Technology. His research covers energy, the environment, climatic change models. He has published more than 18 papers, six monographs and one translation textbook, and has edited several special issues and proceedings.

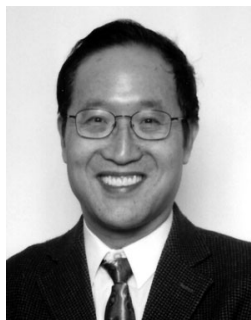

Zhilin $\mathbf{L i}$ is a professor of mathematics, North Carolina State University, USA. He earned his $\mathrm{PhD}$ degree at the University of Washington. His research area is numerical analysis for PDEs, particularly free boundary, moving interface problems. He has published more than 100 papers, one monograph, one textbook, and has edited several special issues and proceedings.

Received: 9 November 2016 Revised: 2 March 2017

Re-revised: 22 May 2017

Accepted: 31 May 2017 